\title{
Serotyping, MLST, and Core Genome MLST Analysis of Salmonella enterica From Different Sources in China During 2004-2019
}

\author{
Shigan Yan ${ }^{1 \dagger}$, Wencheng Zhang ${ }^{1 \dagger}$, Chengyu Li ${ }^{1}$, Xu Liu ${ }^{1}$, Liping Zhu ${ }^{1 *}$, Leilei Chen ${ }^{2 *}$ and \\ Baowei Yang ${ }^{3}$
}

1 Shandong Provincial Key Laboratory of Bioengineering, School of Bioengineering, Qilu University of Technology, Shandong Academy of Sciences, Jinan, China, ${ }^{2}$ Institute of Agro-Food Sciences and Technology, Shandong Academy of Agricultural Sciences, Jinan, China, ${ }^{3}$ College of Food Science and Engineering, Northwest A\&F University, Yangling, China

\section{OPEN ACCESS}

Edited by:

Jun Wang,

Qingdao Agricultural University, China

Reviewed by:

Guojie Cao,

Center for Food Safety and Applied

Nutrition, United States Food and Drug Administration,

United States

Xihong Zhao,

Wuhan Institute of Technology, China

*Correspondence:

Liping Zhu

zhuliping2012@aliyun.com.cn

Leilei Chen

chenleilei8210@163.com

tThese authors have contributed equally to this work

Specialty section:

This article was submitted to

Food Microbiology,

a section of the journal

Frontiers in Microbiology

Received: 31 March 2021

Accepted: 11 August 2021

Published: 16 September 2021

Citation:

Yan S, Zhang W, Li C, Liu X, Zhu L, Chen L and Yang B (2021) Serotyping, MLST, and Core Genome MLST Analysis of Salmonella enterica From Different Sources in China

During 2004-2019.

Front. Microbiol. 12:688614. doi: 10.3389/fmicb.2021.688614
Salmonella enterica (S. enterica) is an important foodborne pathogen, causing food poisoning and human infection, and critically threatening food safety and public health. Salmonella typing is essential for bacterial identification, tracing, epidemiological investigation, and monitoring. Serotyping and multilocus sequence typing (MLST) analysis are standard bacterial typing methods despite the low resolution. Core genome MLST (cgMLST) is a high-resolution molecular typing method based on whole genomic sequencing for accurate bacterial tracing. We investigated $250 \mathrm{~S}$. enterica isolates from poultry, livestock, food, and human sources in nine provinces of China from 2004 to 2019 using serotyping, MLST, and cgMLST analysis. All S. enterica isolates were divided into 36 serovars using slide agglutination. The major serovars in order were Enteritidis (31 isolates), Typhimurium (29 isolates), Mbandaka (23 isolates), and Indiana (22 isolates). All strains were assigned into 43 sequence types (STs) by MLST. Among them, ST11 (31 isolates) was the primary ST. Besides this, a novel ST, ST8016, was identified, and it was different from ST40 by position $317 \mathrm{C} \rightarrow \mathrm{T}$ in dnaN. Furthermore, these 250 isolates were grouped into $185 \mathrm{cgMLST}$ sequence types (cgSTs) by cgMLST. The major cgST was cgST235530 (11 isolates), and only three cgSTs contained isolates from human and other sources, indicating a possibility of cross-species infection. Phylogenetic analysis indicated that most of the same serovar strains were putatively homologous except Saintpaul and Derby due to their multilineage characteristics. In addition, serovar I 4,[5],12:i:- and Typhimurium isolates have similar genomic relatedness on the phylogenetic tree. In conclusion, we sorted out the phenotyping and genotyping diversity of S. enterica isolates in China during 2004-2019 and clarified the temporal and spatial distribution characteristics of Salmonella from different hosts in China in the recent 16 years. These results greatly supplement Salmonella strain resources, genetic information, and traceability typing data; facilitate the typing, traceability, identification, and genetic evolution analysis of Salmonella; and therefore, improve the level of analysis, monitoring, and controlling of foodborne microorganisms in China. 


\section{INTRODUCTION}

Salmonella enterica (S. enterica) is one of the primary foodborne pathogens to cause food poisoning and human infection (Zhang et al., 2015). Foodborne salmonellosis is an important public health concern worldwide, and it annually causes about 115 million infections and 370,000 deaths globally (Seif et al., 2018). The primary sources of $S$. enterica foodborne infection include poultry eggs, meats, and their derived products (Arthur et al., 2008). Therefore, it is crucial to monitor Salmonella from animal food, especially poultry eggs, and their derived food products (Mezal et al., 2014).

Accurate typing and tracing are essential for microbial epidemiological investigation, food safety, and public health. Bacterial typing methods include phenotyping and genotyping. Among them, serotyping and multilocus sequence typing (MLST) are the most frequently used.

Serotyping identification has become the general standard method for Salmonella traceability and phenotypic classification for nearly 100 years. Serotype classification is based on serum agglutination tests of bacterial $\mathrm{O}$ and $\mathrm{H}$ antigens using the White-Kauffmann-Le Minor (WKL) scheme, which is adopted worldwide by public health organizations (Mezal et al., 2014). Up to now, more than 2610 serovars (also serotypes) of Salmonella have been documented globally (Monte et al., 2021). Moreover, Salmonella serotypes usually relate to their host adaptation and virulence, and the change in serotype proportion could reflect the epidemic status, so serotyping plays an essential role in Salmonella surveillance and outbreak investigations (Fierer and Guiney, 2001; Kumar et al., 2009; Dera-tomaszewska, 2012; Barbour et al., 2015). In the past, more attention has been paid to serovars Typhimurium and Enteritidis (Almeida et al., 2018).

In recent years, gene sequencing-based typing assays have been rapidly developed for bacterial tracing with vigorous vitality, including pulsed-field gel electrophoresis, MLST, core genome MLST (cgMLST), whole genome multilocus sequence typing (wgMLST), and whole gene single nucleotide polymorphism (wgSNP) (Liu et al., 2016, 2019; Radomski et al., 2019; Tiba-Casas et al., 2019). Among these molecular typing methods, MLST was developed to establish analytical microorganism typing (Maiden et al., 1998), recognize evolutionary relationships of Salmonella (Achtman et al., 2012; Ashton et al., 2016; Mairi et al., 2020; Zhang et al., 2020), and determine clonal isolate distributions across various environments and hosts (Wang M. et al., 2020; Zhao et al., 2020). In addition, there is a strong correlation between serotypes and sequence types (STs) of Salmonella (Wang X. et al., 2020). However, MLST cannot obtain accurate traceability of Typhimurium and I 4,[5],12:i:- because Salmonella isolates of these two serovars are often divided into the same STs (Possebon et al., 2020).

With the widespread extension of whole genomic sequencing (WGS), WGS-based high-resolution molecular subtyping methods have become popularized in outbreak investigation and bacterial tracing. As a kind of WGS-based subtyping method, cgMLST has high accuracy and can divide strains with minor sequence differences into different cgMLST sequence types (cgSTs), providing a powerful typing approach for molecular epidemiologic investigations. It is most commonly applied for foodborne disease surveillance in the public health area (Yoshida et al., 2016; Vincent et al., 2018; Ben Hassena et al., 2021). cgMLST is proven to be an adequate tool for cluster definition and has become a routine means in many countries and laboratories (Mellmann et al., 2011; Sabat et al., 2017; Simon et al., 2018; Robertson et al., 2019; Wang Y. J. et al., 2020; Hyeon et al., 2021).

In this article, we examine the serotypes, MLST, and cgMLST of $S$. enterica isolates from different sources in nine provinces in China from 2004 to 2019 to investigate their phenotyping and genotyping diversities and genetic relationships.

\section{MATERIALS AND METHODS}

\section{Salmonella enterica Isolates}

A total of $250 \mathrm{~S}$. enterica isolates tested were from different sources in nine provinces in China from 2004 to 2019 except for 2005 and 2013 (Figure 1). The nine provinces, including Guangdong, Guangxi, Fujian, Sichuan, Shaanxi, Henan, Shandong, Shanghai, and Beijing, were major animalbreeding regions in China. The numbers of $S$. enterica isolates from different sources, years, or provinces were not equivalent. The details of these S. enterica isolates are shown in Supplementary Table $\mathbf{1}$. Out of these 250 isolates, 197 were from poultry-derived products, 31 from human, 15 from livestock meat, and 7 from infant nutrition rice formula (food); 16 were isolated in 2004, 9 in 2006, 4 in 2007, 11 in 2008, 31 in 2009, 105 in 2010, 27 in 2011, 13 in 2012, 1 in 2014, 10 in 2015, 5 in 2016, 5 in 2017, 7 in 2018, and 5 in 2019; 13 were separated in Guangdong, 19 in Guangxi, 11 in Fujian, 28 in Sichuan, 67 in Shaanxi, 18 in Henan, 10 in Shandong, 29 in Shanghai, and 55 in Beijing.

Among the 250 S. enterica isolates, 219 strains were isolated from animal products or nutrition rice formula samples. All food samples were immediately homogenized and subjected to Salmonella isolation following the standard protocol as previously described (World Health Organization (WHO), 2003). In short, the sample homogenates were added into selenite cysteine broth and incubated at $35^{\circ} \mathrm{C}$ for $24 \mathrm{~h}$ to selectively enrich Salmonella. Each enriched broth was streaked onto Salmonella Shigella and xylose lysine deoxycholate agar plates and incubated at $37^{\circ} \mathrm{C}$ for $24 \mathrm{~h}$. Presumptive Salmonella colonies were picked and cultured in triple sugar iron agar media and then systematically identified by microbiological, biochemistry, and $16 \mathrm{~S}$ rDNA sequencing analysis to confirm Salmonella strains. Thirty-one Salmonella isolates from humans were isolated and identified by the Beijing Center for Disease Prevention and Control. All identified Salmonella isolates were stored in 25\% $(\mathrm{v} / \mathrm{v})$ glycerol at $-80^{\circ} \mathrm{C}$ in our lab and reproduced periodically.

\section{Serotyping by Slide Agglutination and Prediction by Genome}

Salmonella enterica isolates were cultured in nutrient broth at $37^{\circ} \mathrm{C}$ overnight. A drop of fermentation broth was taken on glass slides to test somatic $\mathrm{O}$ antigen by slide agglutination. Meanwhile, each strain was grown on Swarm agar plates 


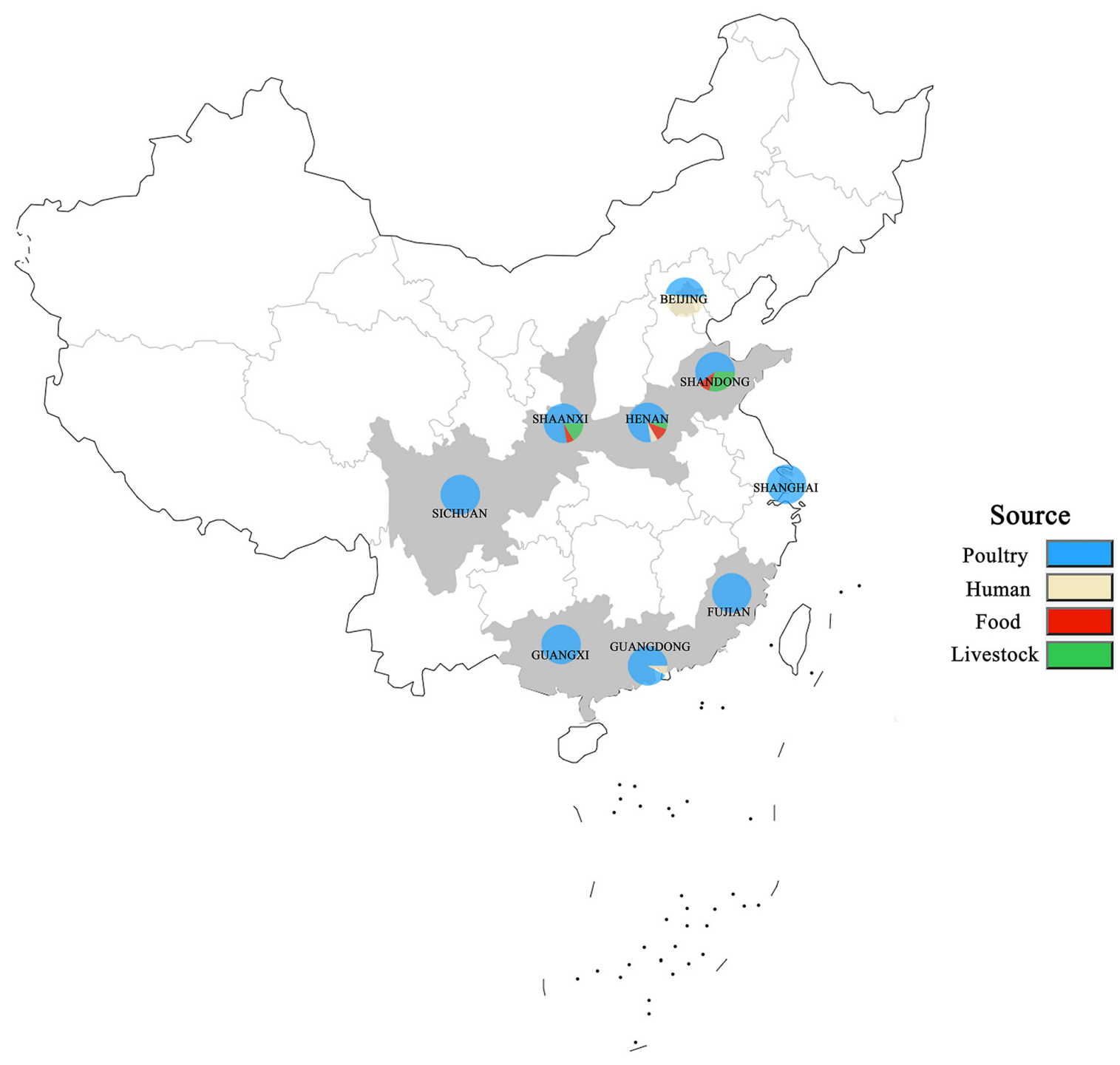

FIGURE 1 | Distribution of S. enterica isolates in China from 2004 to 2019 . The locations where the strains were isolated are shown on the map. The isolates obtained from humans, poultry, livestock, and food are labeled in different colors.

at $37^{\circ} \mathrm{C}$ overnight, and single colonies were picked to test phases 1 and 2 of $\mathrm{H}$ antigens by slide agglutination. Diagnostic sera for Salmonella antigens were purchased from Tianrun Bio-Pharmaceutical Co. (Ningbo, China) and SandA Reagents Lab Ltd. (Bangkok, Thailand). Salmonella serotyping was classified by the WKL scheme. Additionally, $\mathrm{O}$ antigen, $\mathrm{H}$ antigen, and serovars were predicted based on Salmonella genomes using Salmonella In Silico Typing Resource $\left(\right.$ SISTR $\left.^{1}\right)$.

\section{Bacterial Genome Sequencing and Genomic Assembly}

The genomic DNA of each $S$. enterica isolate was extracted with the sodium dodecyl sulfate method using the TIANamp

${ }^{1}$ https://lfz.corefacility.ca/sistr-app/
Bacteria DNA Kit DP302-02 (TIANGEN, China) following the manufacturer's instructions. The extracted genomic DNA quality and integrity were evaluated on $0.5-1 \%$ agarose gels, concentration was measured using a fluorimeter $(\mathrm{MD} 2000 \mathrm{H}$, Biofuture), and purity was determined with a spectrophotometer based on the ratio of $\mathrm{OD}_{260}$ to $\mathrm{OD}_{280}\left(\mathrm{OD}_{260} / \mathrm{OD}_{280} \geq 1.8\right)$. The sequence libraries were constructed using Illumina's Nebnext Ultra DNA Library Prep Kit (NEB, United States). According to different attribute sequences, each sample was assigned with an index code. In brief, the DNA sample was first broken into approximately $350 \mathrm{bp}$ fragments by sonication. After end repair, DNA fragments were ligated head-to-tail to a full-length adaptor for further PCR amplification. The PCR products were purified by the AMPure XP system, the size distribution of the libraries was analyzed by the Agilent bioanalyzer, and quantitative analysis was performed by real-time PCR. 
The genomic DNA of 250 S. enterica isolates was sequenced using the Illumina NovaSeq PE150 platform at Beijing Novogene Bioinformatics Technology Co., Ltd. Considering the influence of low-quality data in the obtained raw sequencing data on the accuracy and reliability of subsequent information analysis, the original data were filtered to obtain the clean data. The specific processing steps included (1) removal of the reads containing low-quality bases (mass value $\leq 20$ ) over a certain percentage (the default was 40\%), (2) removal of reads containing a higher proportion of $\mathrm{N}$ (the default was $10 \%$ ), (3) removal of sequences overlapping with adapters exceeding a certain threshold (the default was $15 \mathrm{bp}$ ) and with fewer than three mismatches, and (4) removal of data that might originate from the host after BLASTing against the host database.

The specific processing steps for genome assembly with clean data included (1) assembling with SOAP de novo software (Li et al., 2008) with different $K$-mers (the default was 107) first and then with the optimal $K$-mer after adjusting other parameters (-d - $\mathrm{u}-\mathrm{R}-\mathrm{F}$, etc.) according to the project type, and the least scaffolds were chosen as the preliminary assembly result; (2) assembling with SPAdes software (Bankevich et al., 2012) with different $K$-mers (the default was 127 ) and then with the optimal $K$-mer according to the project type, and the assembly results as the least scaffolds were obtained; (3) assembling with Abyss software (Simpson et al., 2009) with the 64 nt $K$-mer to obtain the assembly results; (4) using the CISA software to integrate the above three assembly results, and only the assembly results with the least scaffolds were selected; (5) using GapCloser software to fill the gap of preliminary assembly results and remove the same lane pollution by filtering the reads with low sequencing depth (less than 0.35 of the average depth) to obtain the final assembly results; and (6) counting the final assembly result (without fragments below 500 bp) for gene prediction by GeneMarkS software (Besemer et al., 2001).

\section{MLST, cgMLST, and Phylogenetic Analysis}

Salmonella enterica isolates were analyzed based on genomic sequences (clean data) using in silico MLST and cgMLST analysis on the EnteroBase online platform ${ }^{2}$ for Salmonella. Seven housekeeping gene loci, including aroC, dnaN, hemD, hisD, purE, sucA, and thrA, were chosen for MLST analysis of Salmonella. The neighbor-joining tree of $S$. enterica was established based on MLST information.

A Salmonella cgMLST v2 scheme comprising 3002 target loci of Salmonella was employed to analyze cgMLST based on genomic sequences, and neighbor-joining was used to make the dendrograms based on cgMLST information. Salmonella GrapeTree was constructed based on the above cgMLST scheme by EnteroBase GreepTree using NINJA neighborjoining algorithm.

The tanglegram algorithm was applied to compare the MLST and cgMLST phylogenetic trees by placing trees side by side and drawing a straight line (or connector) between corresponding taxa (Scornavacca et al., 2011; Huson and Scornavacca, 2012).

${ }^{2}$ http://enterobase.warwick.ac.uk
Although the algorithm can effectively reduce the number of intersections between connectors, the change of nodes in the evolution of these two types of phylogenetic trees are in the same direction and can lead to multiple short-distance intersections between connectors. If the two types of trees were identical and no connectors crossed, the cluster of phylogenetic tips remains unchanged.

\section{RESULTS}

\section{Serotyping Classification of S. enterica in China During 2004-2019}

The $250 \mathrm{~S}$. enterica strains tested in the study were divided into 36 serotypes by slide agglutination (Figure 2A). Salmonella serovar Enteritidis $(n=31)$ was the most common serotype in China from 2004 to 2019 , followed by Typhimurium $(n=29)$, Mbandaka $(n=23)$, Indiana $(n=22)$, Derby $(n=21)$, Thompson $(n=17)$, Agona $(n=12)$, and I 4,[5],12:i:- $(n=9)$. Senftenberg, Rissen, and Albany accounted for 3.2\% $(n=8)$, respectively. Braenderup and Schwarzengrund possessed seven and six isolates, respectively. Cerro and Corvallis both had five isolates. Blockley, Hadar, Infantis, Meleagridis, Newport, and Saintpaul each encompassed four isolates. Havana and Kentucky both had three isolates. Bovismorbificans, Hvittingfoss, Kottbus, and Stanley each contained two isolates. Anatum, Litchfield, London, Pomona, Potsdam, Tennessee, and Uganda each included one isolate. There were 19 serotypes observed in more than two provinces. Albany, Mbandaka, Enteritidis, I 4,[5],12:i:-, and Typhimurium appeared in three provinces.

The $250 \mathrm{~S}$. enterica isolates were divided into different serotypes based on their sources (Supplementary Table 1). The 197 isolates from poultry belonged to 34 serotypes, among which Typhimurium had the most isolates $(n=24)$, followed in turn by Indiana $(n=20)$, Enteritidis $(n=20)$, Mbandaka $(n=18)$, Derby $(n=17)$, and Thompson $(n=16)$. The 31 isolates from humans belonged to 13 serotypes with Enteritidis accounting for the largest percentage with $32.26 \%(n=10)$. The 15 isolates from livestock contained nine serotypes, among which Derby and Mbandaka each included four stereotypes. In addition, the seven isolates from foods belonged to seven different serotypes.

According to statistics of the isolation years, the number of isolates was different in each year with Typhimurium, Enteritidis, or I 4,[5],12:i:- being the most abundant ones during 2012-2019. Our investigations are similar to those recently reported; Enteritidis and Typhimurium are still major serovars of Salmonella from animal food or humans (Greig and Ravel, 2009; Hendriksen et al., 2011; Yang et al., 2019; Perry et al., 2020; Shen et al., 2020; Xin et al., 2021).

Comparative serotyping analysis of the 250 isolates showed that the serotypes of 245 (98\%) isolates based on slide agglutination were consistent with the genome-based prediction, and only five isolates, QLULP2, QLUY902, QLUY914, QLUY931, and QLUY933, were different. Among them, serotype Typhimurium of QLULP2, QLUY902, QLUY914, and QLUY933 based on slide agglutination was wrongly predicted as I 4,[5],12:i:-, and the serotype I 4,[5],12:i:- of QLUY931 based 


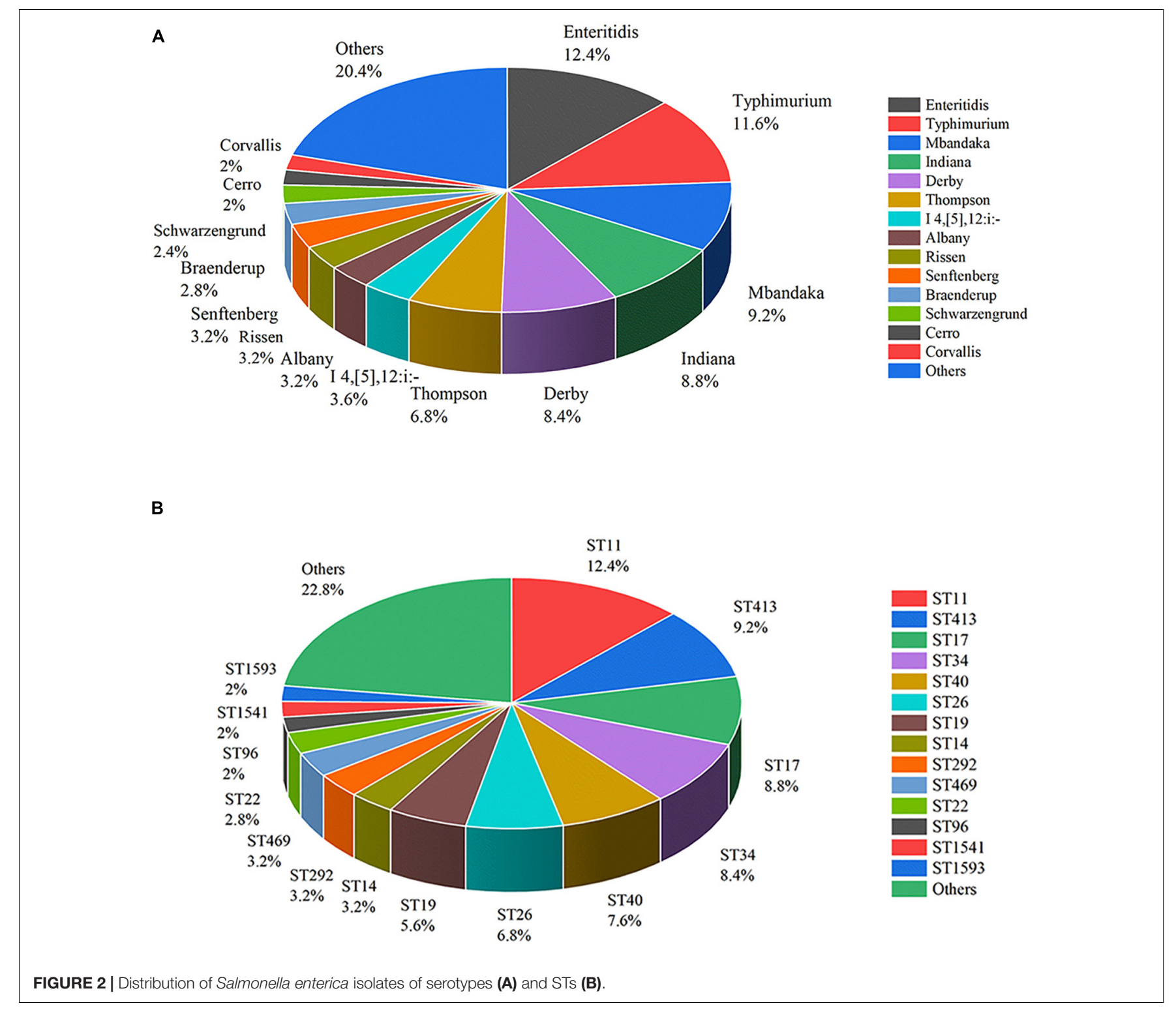

on slide agglutination was wrongly predicted as Typhimurium. It is worth noting that Typhimurium and I 4,[5],12:i:- were rare in genome-based prediction by SISTR in our study. The results proved once again that Salmonella serotype prediction based on the genome is concordant with serovar by the serum agglutination test except Typhimurium and I 4,[5],12:i:-, so WGS-derived serotyping can replace the agglutination assay to some extent and be applied in the typing, traceability, and identification of Salmonella.

\section{Salmonella Draft Genome Analysis}

The genomic reads of $250 \mathrm{~S}$. enterica isolates were stored in the Sequence Read Archive (SRA) of the National Center for Biotechnology Information (NCBI). SRA serial numbers of the submitted 250 strains are shown in Supplementary Table 1.

A total of 9656 ( $\geq 500 \mathrm{bp}$ ) contigs varying from 9 to 92 with an average of 38.62 per genome were generated. The average draft genome size was $4.88 \mathrm{Mb}$, ranging from 4.35 to $5.48 \mathrm{MB}$. Likewise, the average G + C content observed was $52.04 \%$. Functional annotation of all draft genomes predicted an average of 4692 genes, ranging from 4173 to 5334 .

\section{In silico MLST Analysis of S. enterica}

The 250 Salmonella isolates were divided into 43 STs and 37 eBurst groups (eBGs) using in silico MLST. However, ST3134 belonged to neither eBG. ST11 was the most common $(n=31)$, followed in turn by ST413 $(n=23)$, ST17 $(n=22)$, ST34 $(n=21)$, ST40 $(n=19)$, ST26 $(n=17)$, ST19 $(n=14)$, ST14 $(n=8)$, ST292 $(n=8)$, and ST469 $(n=8)$. ST distribution is shown in Figure 2B.

In addition, among the 43 STs identified, a novel ST named ST8016 (QLUY608) was obtained, and it differed from ST40 by 1 $\mathrm{SNP}$ in the dnaN locus at position $317 \mathrm{C} \rightarrow \mathrm{T}$, named dnaN1076, compared to dnaN20). Compared with the ST40 dnaN locus, this mutation occurred in the second codon position, resulting 
in a non-synonymous change from $\mathrm{C}$ to Y. Our findings enrich MLST data of Salmonella, facilitating the typing, traceability, and identification of Salmonella.

Among the 43 STs, 25 STs were observed in more than two provinces and 18 STs in one province. ST17 and ST40 appeared in seven provinces. The results indicate that these two STs were prevalent and had the possibility of transregional infection.

Statistics of the isolation sources revealed that (1) the 31 isolates from humans consisted of 13 different STs, among which ST11 had the highest number of 10 isolates, followed by ST34 ( $n=6)$; (2) the 197 isolates from poultry belonged to 37 STs, among which ST11 had the highest number $(n=22)$, followed in turn by ST413 $(n=21)$, ST17 $(n=16)$, ST40 $(n=16)$, and ST26 $(n=15)$; (3) the 15 isolates from livestock contained 9 STs, among which ST413 had the highest number $(n=4)$; and (4) the 7 isolates from food belonged to 7 different STs.

Statistics of isolation years found that the major ST of every year was different during 2004-2011. ST413 was the major ST in 2004, 2007, and 2011; ST11 was the major ST during 2012-2019 except 2014; and ST34 was the major ST from 2014 to 2019 except 2018. The results showed that the major ST of Salmonella was not constant in every year.

Comparison of MLST and serotyping showed that each ST only comprised one serotype except ST34. Nine isolates of I 4,[5],12:i:- and 12 isolates of Typhimurium belonged to ST34. In addition, four serovars included more than one ST: 29 isolates with serotype Typhimurium contained four STs (ST19, ST34, ST128, and ST1544), 21 isolates of Derby contained three STs (ST40, ST8016, and ST71), 4 isolates of Newport contained 2 STs (ST50 and ST3134), and 6 isolates of Schwarzengrund contained two STs (ST96 and ST241). The results represent that the accuracy of MLST was higher than that of serotyping.

\section{cgMLST Analysis of Salmonella Based on Genomic Sequences}

A total of 3002 target genes were identified from the S. enterica genome using the EnteroBase cgMLST module. The cgMLST analysis of S. enterica isolates is shown in Supplementary Table 1. The cgMLST analysis revealed that the 250 Salmonella isolates were grouped into $185 \mathrm{cgSTs}$, all of which contained only one serotype. Among them, 243 isolates belonged to 182 novel cgSTs, and 7 belonged to three known cgSTs. Among the $185 \mathrm{cgSTs}$, cgST235530 contained the most abundant isolates, followed by cgST217495 $(n=6)$ and cgST234930 $(n=6)$. We comprehensively analyzed the WGS-based genotypes of Salmonella in China in the past 16 years and found many novel cgSTs, which enriched the genotype data resources and promoted the development of traceability level of Salmonella.

The relationship of cgST with the years, regions, and sources of isolation is shown in Table 1. Among the $185 \mathrm{cgSTs}, 33 \mathrm{cgSTs}$ contained more than two isolates, and $15 \mathrm{cgSTs}$ contained strains from different years, provinces, or sources of isolation. In detail, $12 \mathrm{cgST}$ isolates were from multiple provinces, $12 \mathrm{cgST}$ from different years, and $5 \mathrm{cgST}$ from different sources, representing the possibility of cross-outbreaking in different locations, hosts, or years. cgST236211, cgST236321, and cgST236324 contained
TABLE 1 | Salmonella enterica cgSTs from different years, provinces, or sources of isolation.

\begin{tabular}{lcccc}
\hline cgST & Numbers & Source & Province & Years \\
\hline cgST42868 & 5 & Poultry & Shaanxi, Fujian & 2010,2011 \\
cgST217495 & 6 & Poultry & Beijing, Shaanxi & 2010,2012 \\
cgST234925 & 3 & Poultry & Sichuan, Fujian & 2010 \\
cgST234927 & 3 & Poultry & Sichuan, Shaanxi & 2006,2010 \\
cgST234930 & 6 & Poultry, food & Sichuan, Shaanxi & 2006,2007, \\
cgST234932 & 3 & Poultry & Sichuan, Fujian & 2010 \\
cgST235529 & 2 & Poultry, food & Sichuan, Shaanxi & 2006,2010 \\
cgST235530 & 11 & Poultry & Shaanxi, Guangdong & 2010,2011 \\
cgST235678 & 2 & Poultry & Sichuan, Shaanxi & 2010,2011 \\
cgST235686 & 2 & Poultry & Guangxi, Shaanxi & 2010,2011 \\
cgST235712 & 2 & Poultry & Shaanxi, Shanghai & 2006 \\
cgST235797 & 2 & Poultry & Guangdong, Shaanxi & 2007,2011 \\
cgST236211 & 2 & Poultry, human & Guangdong & 2010 \\
cgST236321 & 2 & Poultry, human & Beijing & 2008,2012 \\
cgST236324 & 2 & Poultry, human & Beijing & 2009,2012 \\
\hline
\end{tabular}

multiple isolates from human and other sources, indicating a possibility of cross-species infection. In addition, 152 strains had unique cgSTs in this study.

A neighbor-joining tree was generated based on the 250 strains' cgMLST information (Figure 3). Many serovars formed serovar-specific clades in this tree, suggesting that the same serovars were putatively homologous except that isolates of Saintpaul, Derby, and I 4,[5],12:i:- were heterologous. To further investigate the relationship of Saintpaul, Derby, and I 4,[5],12:i:isolates, the GrapeTrees of each serovar were constructed based on cgMLST information (Figure 4). In the GrapeTrees, Saintpaul contained four isolates belonging to two clades with a long genetic distance, representing that Saintpaul was putatively polyphyletic (Figure 4A). Similarly, Derby contained 21 isolates that did not wholly cluster together. QLUY614 (cgST236253) had a long genetic distance to the other 20 isolates, suggesting Derby was putatively polyphyletic, too (Figure 4B). Differently, the nine isolates of serovar I 4,[5],12:i:- had close genetic distances in the GrapeTree even though they did not completely cluster together (Figure 4C). In a word, isolates of Saintpaul and Derby were putatively polyphyletic and characterized by multilineages (Yin et al., 2020).

Additionally, I 4,[5],12:i:- and Typhimurium in a similar genetic distance may have a near genetic relationship (Figure 3). To further investigate the relatedness of I 4,[5],12:i:- and Typhimurium, their GrapeTree was constructed based on cgMLST information of serovar I 4,[5],12:i:- $(n=9)$ and Typhimurium $(n=28)$ (Figure 5). In the GrapeTree, Typhimurium isolates were clustered into eight highly clonal clades and four highly clonal clusters (clusters 1-4), and nine isolates of I 4,[5],12:i:- and 12 isolates of Typhimurium were clustered into cluster 4 . The low homologous diversity within I 4,[5],12:i:- and Typhimurium in combination with the above cgST tree on coherent clades indicate that these two serovars might have similar genomic sequences. 


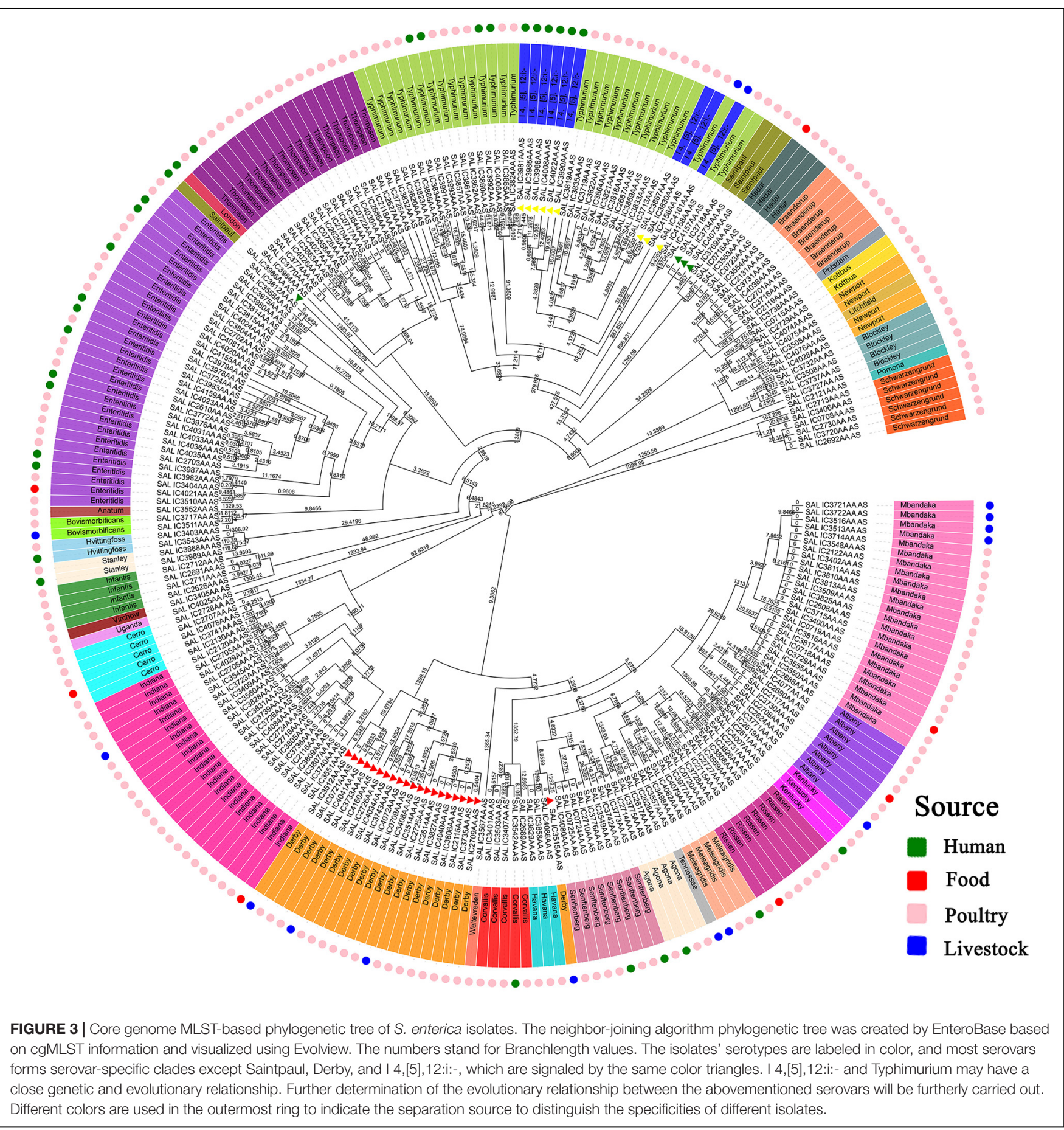

In addition, only three isolates from patients were in the same genetic position with other strains in the evolutionary tree. As mentioned, isolates of cgST236211, cgST236321, and cgST236324 were in the same genetic position in the tree: QLULY5 (human, Beijing, 2009) with QLULR4 (poultry, Beijing, 2012) of Thompson; QLULY1 (human, Beijing, 2008) with QLULR2 (poultry, Beijing, 2012) of Senftenberg; and QLULN4 (human, Guangdong, 2010) with QLUY510 (poultry, Guangdong, 2010) of Hvittingfoss. There was a strong homology among these isolates and a possibility of cross-species infection in patients although significant differences existed in some isolates from different years of isolation.

Analysis of isolates' clustering position in the evolutionary tree showed isolates of Hadar, I 4,[5],12:i:-, Saintpaul, Kentucky, and Mbandaka had a strong correlation between genetic position and source (Figure 3). Among the three Kentucky isolates, QLULA8 (Guangxi, 2010) and QLULA5 (Beijing, 2010) isolates from poultry were putatively homologous and had a long genetic 


\section{A}

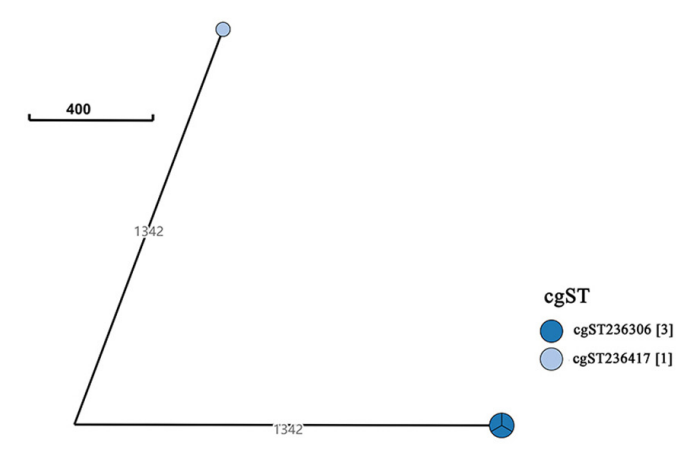

B
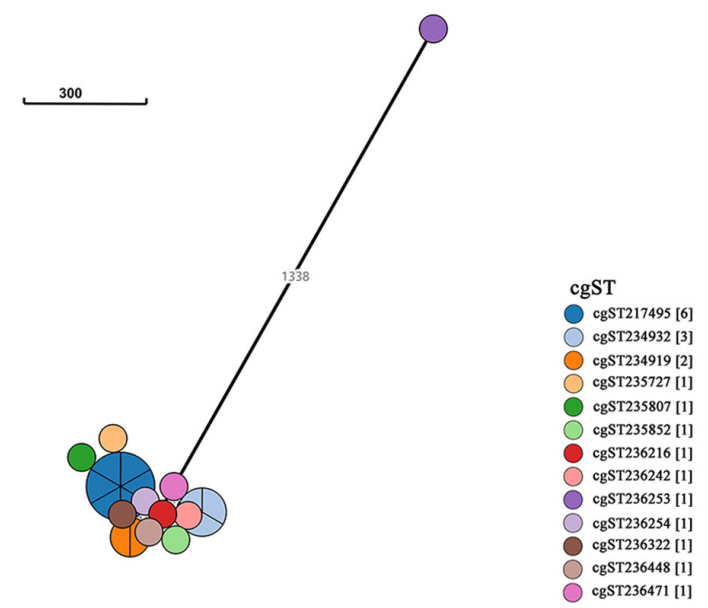

C

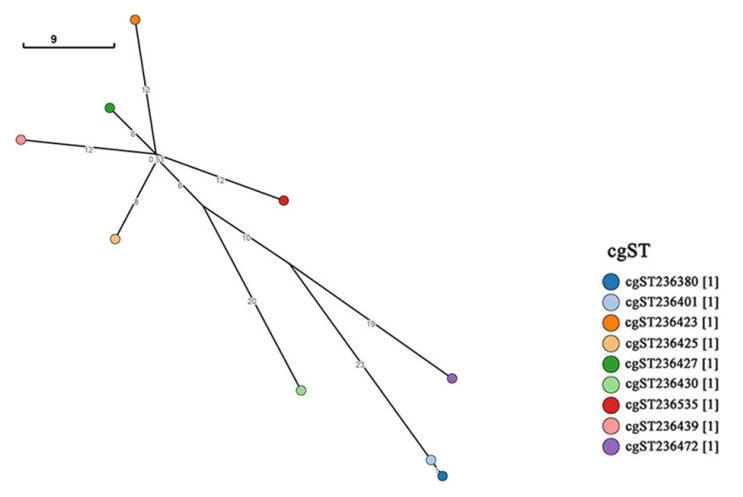

FIGURE 4 | GrapeTree of Salmonella serovar Saintpaul, Derby, and I 4,[5],12:i:- isolates. GrapeTree constructed based on the 3002 core genes by EnteroBase GrapeTree using NINJA neighbor-joining algorithm. (A) GrapeTree among the four serovar Saintpaul isolates, where every node means different cgST and the line between two nodes indicates a long distance ( $\geq 1000)$; (B) GrapeTree of the 21 Derby and $1 \mathrm{cgST}$ (cgST236253) isolates with a long genetic distance ( $\geq 1000$ ) to other cgSTs; (C) GrapeTree of the nine serovar I 4,[5],12:i:- and nine cgST isolates with nodes indicating they all have a relatively close genetic distance $(\leq 100)$. distance to the isolate QLUF123 (Beijing, 2018) from patients. What is more, among the 13 Mbandaka isolates, four from livestock were in the same genetic position in the evolutionary tree with high homology and had a long genetic distance to other isolates of different sources. Obviously, serovar I 4,[5],12:i:isolates from the same hosts were at an adjacent genetic position in the evolutionary tree.

\section{Major Serovars and cgSTs of Salmonella Isolates From Different Sources, Years, and Provinces}

A total of 25 major cgSTs and 26 major serovars appeared among the 250 Salmonella isolates from different sources in nine provinces during 2004-2019. Among them, four major serovars from livestock in Fujian province in 2007 and 2011 were clustered in major cgSTs. In detail, 11 major cgSTs belonged to major serovars. For example, four isolates of cgST236240 in 2004 belonged to Mbandaka, and the major serovars in 2004 were Derby $(n=4)$ and Mbandaka $(n=4)$. Obviously, the major serovar Typhimurium clustered with 19 isolates in 2009, but the major cgST was cgST236385 ( $n=3$, Havana). Meanwhile, the major serovar from poultry was Typhimurium $(n=28)$, and the major cgST was cgST235530 $(n=11$, Mbandaka). The 28 Typhimurium isolates were divided into 26 different cgSTs and seven isolates from food belonged to seven serovars and seven cgSTs. The major serotype was always Typhimurium, Enteritidis, or I 4,[5],12:i:- during 2015-2019. However, these three serovar isolates possessed different cgSTs. Major cgSTs and major serovars of Salmonella isolates from different sources in different years and provinces are shown in Table 2.

\section{Comparative Analysis of MLST and cgMLST Within S. enterica Isolates}

Identification of all loci present within Salmonella isolates was performed using MLST and cgMLST schemes. The 250 S. enterica isolates in China from 2004 to 2019 were used to generate a tanglegram for a visual comparison of cgMLST and MLST (Figure 6). Most of the straight lines were parallel, and only some minor straight lines crossed with others, indicating that the majority of STs were parallel to cgSTs. This phenomenon may give rise by differences between the located deeper internal nodes within these two phylogenies. By comparison, most of the isolates were grouped into the same clusters by MLST and cgMLST.

Further analysis shows that the crosslines were mainly due to the differences in the genetic relationships between some different serovar isolates in the evolutionary tree established by MLST and cgMLST. By comparison, the following serovar isolates had close genetic relationships in the MLST-based neighbor-joining tree but not in the cgMLST-based neighborjoining tree: (1) 1 isolate of Virchow (QLUY603) with 5 Corvallis isolates, (2) 1 isolate of Weltevreden (QLULO6) with all 23 Mbandaka isolates and 8 Albany isolates, (3) 3 isolates of Kentucky with all 8 Rissen isolates, (4) f isolates of Cero with 1 Saintpaul isolate (QLUF115), and (5) 5 isolates of Saintpaul and all 31 Enteritidis isolates. Although MLST and cgMLST clustered two isolates of Stanley (QLUF119, QLUZ101) and 


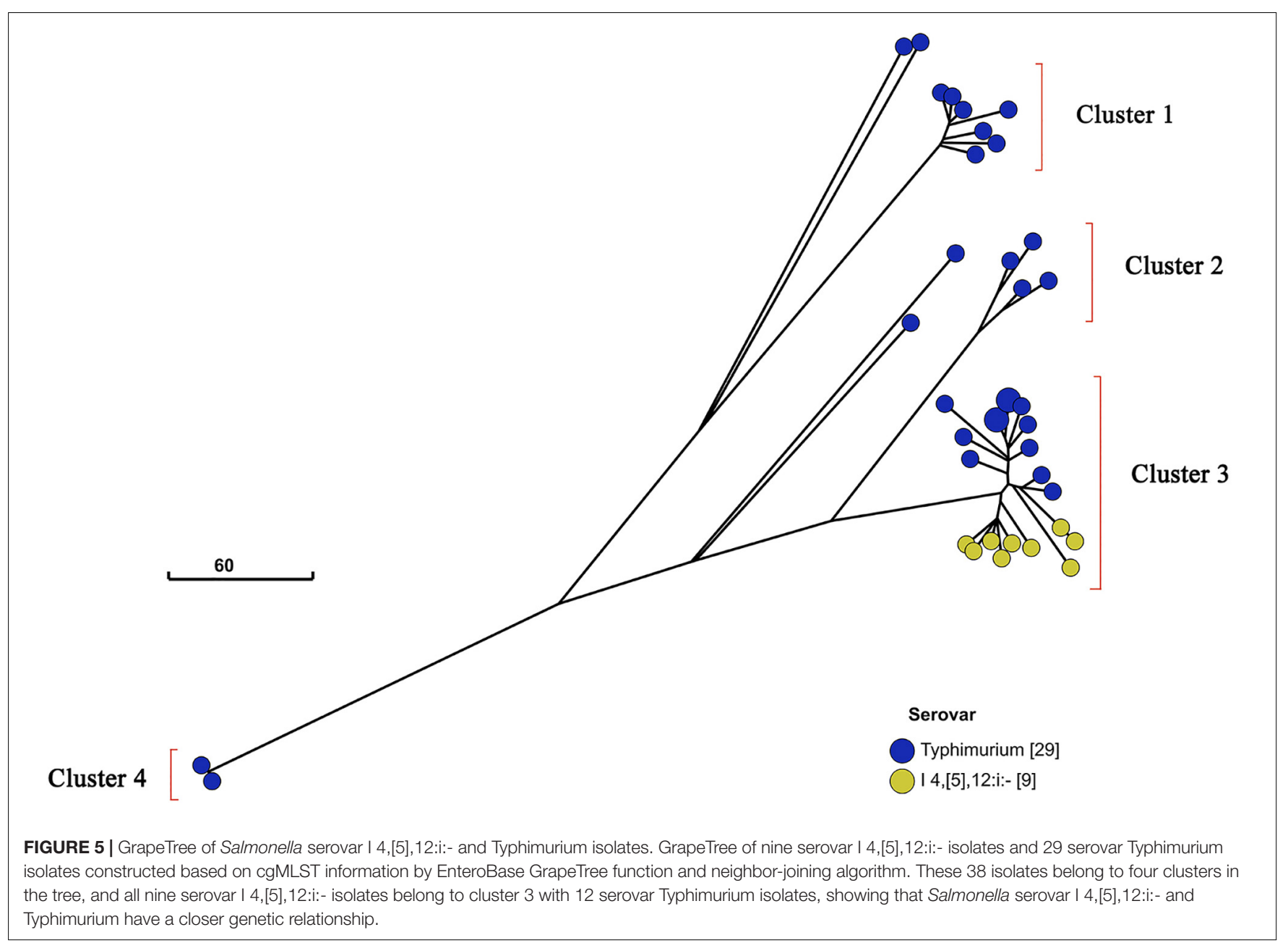

two isolates of Hvittingfoss (QLULN4, QLUY510) together, the MLST-based neighbor-joining tree indicated one Virchow isolate (QLUY603) is closely related with five Corvallis isolates, and the cgMLST-based neighbor-joining tree placed them at a longer genetic distance. However, the majority of Salmonella isolates were grouped into the same clusters by both MLST and cgMLST. Obviously, the evolutionary tree by cgMLST was more detailed than that by MLST.

\section{DISCUSSION}

In recent years, with increasing demand for food safety, the detection of foodborne Salmonella becomes greatly important, and Salmonella from food is tested more intensively worldwide (Yang et al., 2019). Salmonella typing is essential for microbial determination, epidemiological investigation, and outbreak tracing. Serotyping and MLST are the gold standard bacterial typing methods. However, they both could not accurately trace because they are relatively low-resolution. Thus, it is significant to establish high-resolution and reliable subtyping methods for tracing epidemic strains in pathogenic outbreaks (Jolley et al., 2012).
Serotyping by slide agglutination has been widely adopted for Salmonella classification for nearly 100 years. In this study, we serotyped 250 Salmonella isolates from different sources in China in the past 16 years, and the results show all strains tested in the study were divided into 36 serotypes, and Salmonella serovars Enteritidis and Typhimurium were still the major serovars, which is similar to a recent report (Perry et al., 2020; Shen et al., 2020). Enteritidis is frequently isolated from animal food globally and is one of the most common serovars associated with human salmonellosis (Greig and Ravel, 2009; Hendriksen et al., 2011; Yang et al., 2019).

However, serotyping by slide agglutination has some shortcomings, such as being low-resolution, expensive, and time-consuming. In addition, this serological test requires well-trained personnel to operate it as well as high-quality sera; otherwise, it is prone to error in serotype determination because of artificial discrimination of differences in agglutination profiles and the incomplete phenomenon of $\mathrm{H}$ antigen expression (Zhou et al., 2020). However, it does not mean that serotyping will be rapidly replaced because it has become a traditional microbial phenotyping classification method by microorganists and public health organizations. Therefore, it will still play a fundamental role in bacterial tracing in the near future. 
TABLE 2 | Major cgSTs and major serovars of S. enterica isolates.

\begin{tabular}{|c|c|c|c|}
\hline & Number of strains & Major cgSTs (no., serovar) & Major serovars (no.) \\
\hline \multicolumn{4}{|l|}{ Year } \\
\hline 2004 & 16 & cgST236240 (4, Mbandaka) & Derby (4), Mbandaka (4) \\
\hline 2006 & 9 & cgST235712 (2, Infantis) & Infantis (2), Thompson (2) \\
\hline 2007 & 4 & cgST234930 (2, Mbandaka) & Mbandaka (2) \\
\hline 2008 & 11 & cgST236457 (2, Meleagridis) & Corvallis (3) \\
\hline 2009 & 32 & cgST236385 (3, Havana) & Typhimurium (19) \\
\hline \multirow[t]{3}{*}{2010} & 105 & cgST42868 (4, Braenderup) & Indiana (12) \\
\hline & & cgST234918 (4, Schwarzengrund) & \\
\hline & & cgST234928 (4, Senftenberg) & \\
\hline 2011 & 27 & cgST235530 (9, Mbandaka) & Mbandaka (9) \\
\hline 2012 & 13 & cgST217495 (4, Derby) & Enteritidis (6) \\
\hline 2014 & 1 & - & - \\
\hline 2015 & 10 & - & Typhimurium (4) \\
\hline 2016 & 5 & - & Enteritidis (2), I 4,[5],12:i:- (2) \\
\hline 2017 & 5 & - & Enteritidis (2), I 4,[5],12:i:- (2) \\
\hline 2018 & 7 & - & Enteritidis (2), Typhimurium (2) \\
\hline 2019 & 5 & - & Enteritidis (2), I 4,[5],12:i:- (2) \\
\hline \multicolumn{4}{|l|}{ Province } \\
\hline Beijing & 55 & cgST217495 (3, Derby) & Enteritidis (15) \\
\hline Fujian & 11 & cgST234918 (4, Schwarzengrund) & Schwarzengrund (4) \\
\hline \multirow[t]{3}{*}{ Guangdong } & 13 & cgST236218 (2, Corvallis) & Mbandaka (3) \\
\hline & & cgST236211 (2, Hvittingfoss) & \\
\hline & & cgST235530 (2, Mbandaka) & \\
\hline \multirow[t]{4}{*}{ Guangxi } & 19 & cgST235719 (2, Cerro) & Albany (3) \\
\hline & & cgST234919 (2, Derby) & Cerro (3) \\
\hline & & cgST236457 (2, Meleagridis) & Newport (3) \\
\hline & & cgST236458 (2, Newport) & \\
\hline Henan & 18 & - & Enteritidis (3) \\
\hline Shandong & 10 & cgST236436 (2, Enteritidis) & Enteritidis (5) \\
\hline Shanghai & 29 & cgST236385 (3, Havana) & Typhimurium (19) \\
\hline Shaanxi & 67 & cgST234930 (9, Mbandaka) & Mbandaka (18) \\
\hline Sichuan & 28 & cgST234928 (4, Senftenberg) & Thompson (5) \\
\hline \multicolumn{4}{|l|}{ Source } \\
\hline Food & 7 & - & - \\
\hline Human & 31 & - & Enteritidis (10) \\
\hline Livestock & 15 & cgST236240 (4, Mbandaka) & Mbandaka (4) \\
\hline Poultry & 197 & cgST235530 (11, Mbandaka) & Typhimurium (28) \\
\hline
\end{tabular}

"-" means no major serovars or major cgSTs.

We also validated the serotyping by agglutination assay using SISTR prediction based on genomic sequences. The two methods are highly consistent with a consistency rate of $98 \%$, which is similar to recent reports (Robertson et al., 2018; Uelze et al., 2019; Lyu et al., 2021). However, SISTR has a low identification for Typhimurium and I 4,[5],12:i:- in our research. This situation may be because SISTR only predicts serotypes based on related coding genes, and the results of slide agglutination tests are affected by many factors, such as gene mutation or inexpression.

Facing potential food safety risks, serological misidentification may endanger underestimating the occurrence of certain Salmonella serotypes outbreaks. The serotype prediction based on nucleic acid sequences plays an essential role in serotype validation. Therefore, it is essential to validate the slide agglutination assay. Several methods have been developed to predict serotypes based on nucleic acid sequences (Achtman et al., 2012; Inouye et al., 2014; Zhang et al., 2019). MLST is attractive because of being generated with easily replicated protocols and correlated well with the majority of lineages and serovars by means of eBGs. The advantages of MLST are more uniform, well relative with serotypes, and convenient to communicate by databases (Kimura, 2018).

The 250 Salmonella isolates were divided into 43 STs using in silico MLST with ST11 being the most abundant ST $(n=31)$. Among the 43 STs identified, a novel ST, ST8016 (QLUY608), differs from ST40 by $1 \mathrm{SNP}$ in the dnaN locus at position 317 (C $\rightarrow$ T, named dnaN1076, compared with dnaN20). According to MLST typing, ST34 contains I 4, [5],12:i:- and 


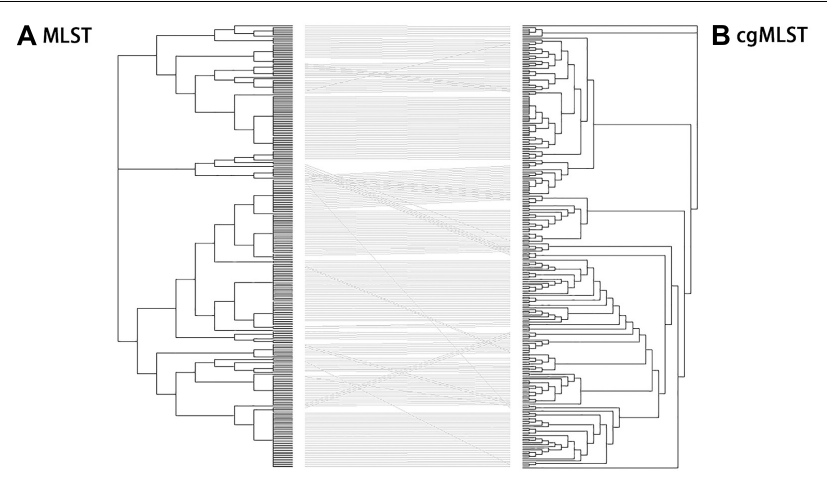

FIGURE 6 | Comparison of phylogenies by MLST (A) and cgMLST (B) based on genomic sequences of 250 Salmonella isolates. Tanglegram linking tips with the same label to each other via a straight line produced within dendroscope 3 of 250 Salmonella isolates. By comparing of $\mathrm{N}-\mathrm{J}$ trees by MLST and cgMLST, clustering in the two trees was mostly congruent although a few sections show several inversions. This led to some isolates being clustered at the edge of one tree, moving to the center of the other. In addition, the tree by cgMLST was more detailed than that by MLST.

Typhimurium. The method cannot distinguish I 4,[5],12:i:- from Typhimurium with the same STs because of similar genomic sequences. Furthermore, a GrapeTree was constructed using the genomic sequences of all isolates of I 4,[5],12:i:- $(n=9)$ and Typhimurium $(n=29)$. The results show that the isolates of the two serovars could not be completely clustered together: only nine isolates of I 4,[5],12:i:- and 12 isolates of Typhimurium belong to the same cluster and are in similar genetic positions on the evolutionary tree (Figure 5). However, the MLST approach may cause two main issues to predict serotype using SISTR: (1) a few STs do not have a serovar designation in the MLST database and (2) the unexpected identification of novel STs.

Molecular typing for Salmonella of the same serovars is essential in outbreak investigation and bacterial epidemiology. The increasing availability of analytical approaches for whole genome-based subtyping will continue to fuel the adoption of genomics in the context of epidemiological investigations. With the reduction of sequencing cost and the promotion of genome sequencing, a large amount of bacterial genome data has been generated, and it has become routine to trace microorganisms based on bacterial genomic sequences. Molecular typing methods based on WGS with high resolution and outstanding accuracy are used as routine bacterial trace analysis methods. Each has different advantages and drawbacks that determine its applicability and limitations. In recent years, cgMLST, wgMLST, and SNP have become innovative tracing tools (García-Soto et al., 2020; Gu et al., 2020; Monte et al., 2021). cgMLST well correlates with time and regions of bacterial isolates. Microorganism epidemiological investigation results found that the main pathogens may probably change during outbreaking, and these will be largely muted by conventional low-resolution typing methods (Quick et al., 2015). In this study, cgMLST can distinguish the differences and confirm the extremely minute association of these strains of major serotypes. Although they have been grouped into Enteritidis, Typhimurium, and I 4,[5],12:i:- since 2015, we judged the prevalent Salmonella was not due to continuous spread of individual strains.

It is worth noting that the traceability is influenced by the use of different cgMLST classification schemes (Li et al., 2008). In several cgMLST scheme protocols, 3002 core loci cgMLST schemes of Salmonella promoted in EnteroBase have been widely accepted because of applying the default settings of loci and alleles most likely to be used by many microbiologists to obtain accordant and accurate applications across laboratories and jurisdictions. EnteroBase is well adopted because it covers a large number of isolates' genomic sequences, and the fixed typing scheme makes the cgMLST results truly comparable among different researchers. EnteroBase covers more than 270,000 Salmonella genomic sequences globally and facilitates cgMLST analysis of Salmonella. The convenience and pertinence of data collection and collation provide strong data support for pathogen evolution analysis. The fixed typing scheme makes it easy to explore the homologous relationship of individual isolates in the database and to invoke more isolates with certain characteristics. Only by retrieving the isolate information, including time, country or region of isolation, serotype, and MLST and cgMLST typing results of the isolate, could the isolate's relevant information be obtained. The availability of a web-based analysis platform enables users to conduct cgMLST analysis with minimal local hardware requirements. cgMLST analysis based on thousands of genomic alleles has a higher typing accuracy than MLST. The former was verified to be a more realistic reflection of the evolutionary relationship within the species (Ruppitsch et al., 2015).

High-resolution molecular methods for Salmonella typing are not replacing MLST because MLST is relatively convenient for establishing a good relationship with serovars (Kimura, 2018). Comparing evolutionary trees by MLST and cgMLST, the two methods have similar clustering results. However, cgMLST can further distinguish even minor differences between isolates, and the clustering is more detailed. Compared with cgMLST, MLST is generally more discriminatory, but it cannot provide a satisfactory resolution for public health surveillance. Our study also indicates cgMLST is significantly more accurate than MLST, no cgST contains two or more serovars, even I 4,[5],12:i:and Typhimurium.

According to the evolutionary analysis, the isolates representing the most serovars formed serovar-specific clades in our established neighbor-joining tree except Saintpaul, I 4,[5],12:i:-, and Derby. To explore the genetic and evolutionary relationship among these three serotypes, we further constructed a GrapeTree of the isolates of the three serovars (Figure 4). Only Saintpaul and Derby have multilineages (Yin et al., 2020).

Among the three typing methods, serotyping showed the lowest resolution, and cgMLST had the highest accuracy. Serotyping was based on reactions of antisera to the lipopolysaccharide and flagellar antigens; otherwise, it did not reflect the genetic relatedness between serovars; MLST, based on seven housekeeping genes, was an accurate, reliable typing method, well suited to routine microbial surveillance; WGSbased cgMLST could greatly improve the accuracy of typing and was convenient to share or compare across international 
labs, so it should be developed in the traceability typing of microorganisms with vitality in the future.

\section{CONCLUSION}

We investigated $250 \mathrm{~S}$. enterica isolates from China during 2004-2019 using serotyping, MLST, and cgMLST. All the Salmonella strains were divided into 36 serovars, 43 STs by MLST, and $185 \mathrm{cgST}$ s by cgMLST. We found the prevalent serovars, STs, and cgSTs of Salmonella from different years, regions, and host sources. In addition, we also discovered a novel ST and 182 novel cgSTs. This article clarifies the temporal and spatial distribution characteristics of phenotyping and genotyping diversity of $S$. enterica isolates in China in the recent 16 years. Our results supplement the strain resources, genetic information, and typing data of Salmonella; benefit the typing, traceability, determination, and genetic evolution analysis of Salmonella; and therefore, promote the level of analysis, monitoring, and prevention and controlling of Salmonella in China.

\section{DATA AVAILABILITY STATEMENT}

The datasets presented in this study can be found in online repositories. The names of the repository/repositories and accession number(s) can be found in the article/ Supplementary Material.

\section{AUTHOR CONTRIBUTIONS}

SY, WZ, and LZ were major contributors in writing the manuscript, and conceived and designed the study. SY, LC, and

\section{REFERENCES}

Achtman, M., Wain, J., Weill, F. X., Nair, S., Zhou, Z., Sangal, V., et al. (2012). Multilocus sequence typing as a replacement for serotyping in Salmonella enterica. PLoS Pathog. 8:e1002776. doi: 10.1371/journal.ppat.1002776

Almeida, F., Amanda, A. S., Marta, I. C. M., Dália dos, P. R., Alessandro De, M. V., Yan, L., et al. (2018). Phylogenetic and antimicrobial resistance gene analysis of Salmonella Typhimurium strains isolated in Brazil by whole genome sequencing. PLoS One 13:e0201882. doi: 10.1371/journal.pone.0201882

Arthur, T. M., Brichta-harhay, D. M., Bosilevac, J. M., Guerini, M. N., Kalchayanand, N., Wells, J. E., et al. (2008). Prevalence and characterization of Salmonella in bovine lymph nodes potentially destined for use in ground beef. J. Food Prot. 71, 1685-1688. doi: 10.4315/0362-028x-71.8.1685

Ashton, P. M., Nair, S., Peters, T. M., Bale, J. A., Powell, D. G., Painset, A., et al. (2016). Identification of Salmonella for public health surveillance using whole genome sequencing. PeerJ. 4:e1752. doi: 10.7717/peerj.1752

Bankevich, A., Nurk, S., Antipov, D., Gurevich, A. A., Dvorkin, M., Kulikov, A. S., et al. (2012). SPAdes: a new genome assembly algorithm and its applications to single-cell sequencing. J. Comput. Biol. 19, 455-477. doi: 10.1089/cmb.2012. 0021

Barbour, E. K., Ayyash, D. B., Alturkistni, W., Alyahiby, A., Yaghmoor, S., Yousef, J., et al. (2015). Impact of sporadic reporting of poultry Salmonella serovars from selected developing countries. J. Infect.Dev. Ctries. 9, 1-7. doi: 10.3855/ jidc. 5065
BY contributed materials and resources. CL and XL worked for slide agglutination. All authors read and approved the final manuscript.

\section{FUNDING}

This work was funded by the National Key Research and Development Program of China (Grant Number 2017YFC1601400), the Natural Science Foundation of Shandong province (ZR2018LC003), and the Key Research and Development Program of Shandong Province (Grant Numbers 2019GNC106154 and 2014GSF120006).

\section{ACKNOWLEDGMENTS}

We thank Qian Chen and Yuanyuan Zhang of Beijing Center for Diseases Prevention and Control for collecting many Salmonella enterica isolates from humans.

\section{SUPPLEMENTARY MATERIAL}

The Supplementary Material for this article can be found online at: https://www.frontiersin.org/articles/10.3389/fmicb. 2021.688614/full\#supplementary-material

Supplementary Table 1| Background information, draft genome, serovars, and genotypes of 250 Salmonella enterica isolates. Comment: assembly barcode was obtained by submitting the raw genome data into EnteroBase, and the replacement strain name played an identification role in the established evolutionary tree. SRA numbers were obtained from the NCBI SRA. It is important to note that prefixes such as "QLUL" were added to the strain's name after submission to EnteroBase in order to obviously distinguish it, so there may be some bias from the SRA database strain names.

Ben Hassena, A., Haendiges, J., Zormati, S., Guermazi, S., Gdoura, R., GonzalezEscalona, N., et al. (2021). Virulence and resistance genes profiles and clonal relationships of non-typhoidal food-borne Salmonella strains isolated in Tunisia by whole genome sequencing. Int. J. Food Microbiol. 337:108941. doi: 10.1016/j.ijfoodmicro.2020.108941

Besemer, J., Alexandre, L., and Mark, B. (2001). GeneMarkS: a self-training method for prediction of gene starts in microbial genomes. Implications for finding sequence motifs in regulatory regions. Nucleic Acids Res. 29, 2607-2618. doi: $10.1093 /$ nar/29.12.2607

Dera-tomaszewska, B. (2012). Salmonella serovars isolated for the first time in poland 1995-2007. Int. J. Occup. Med. Environ. Health 25, 294-303. doi: 10. 2478/S13382-012-0038-2

Fierer, J., and Guiney, D. G. (2001). Diverse virulence traits underlying different clinical outcomes of Salmonella infection. J. Clin. Invest. 107, 775-780. doi: 10.1172/JCI12561

García-Soto, S., Abdel-Glil, M. Y., Tomaso, H., Linde, J., and Methner, U. (2020). Emergence of multidrug-resistant Salmonella enterica subspecies enterica serovar infantis of multilocus sequence type 2283 in German broiler farms. Front. Microbiol. 11:1741. doi: 10.3389/fmicb.2020.01741

Greig, J. D., and Ravel, A. (2009). Analysis of foodborne outbreak data reported internationally for source attribution. Int. J. Food Microbiol. 130, 77-87. doi: 10.1016/j.ijfoodmicro.2008.12.031

Gu, D., Wang, Z., Tian, Y., Kang, X., Meng, C., Chen, X., et al. (2020). Prevalence of Salmonella isolates and their distribution based on whole-genome sequence in 
a chicken slaughterhouse in Jiangsu, China. Front. Vet. Sci. 7:29. doi: 10.3389/ fvets.2020.00029

Hendriksen, R. S., Vieira, A. R., Karlsmose, S., Lo Fo Wong, D. M. A., Jensen, A. B., Wegener, H. C., et al. (2011). Global monitoring of Salmonella serovar distribution from the world health organization global foodborne infections network country data bank: results of quality assured laboratories from 2001 to 2007. Foodborne Pathog. Dis. 8, 887-900. doi: 10.1089/fpd.2010.0787

Huson, D. H., and Scornavacca, C. (2012). Dendroscope 3: an interactive tool for rooted phylogenetic trees and networks. Syst. Biol. 61, 1061-1067. doi: 10.1093/ sysbio/sys 062

Hyeon, J. Y., Li, S. T., Mann, D. A., Zhang, S. K., Kim, K. J., Lee, D. H., et al. (2021). Whole-genome sequencing analysis of Salmonella enterica serotype enteritidis isolated from poultry sources in South Korea, 2010-2017. Pathogens 10:45. doi: $10.3390 /$ pathogens 10010045

Inouye, M., Dashnow, H., Raven, L. A., Schultz, M. B., Pope, B. J., Tomita, T., et al. (2014). SRST2: rapid genomic surveillance for public health and hospital microbiology labs. Genome Med. 6:90. doi: 10.1186/s13073-014-0090-6

Jolley, K. A., Bliss, C. M., Bennett, J. S., Bratcher, H. B., Brehony, C., Colles, F. M., et al. (2012). Ribosomal multilocus sequence typing: universal characterization of bacteria from domain to strain. Microbiology 158(Pt 4), 1005-1015. doi: 10.1099/mic.0.055459-0

Kimura, B. (2018). Will the emergence of core genome MLST end the role of in silico MLST? Food Microbiol. 75, 28-36.

Kumar, Y., Sharma, A., Sehgal, R., Kumar, S., Paratyphi, S. C., Worthington, S., et al. (2009). Distribution trends of Salmonella serovars in India (2001-2005). Trans. R. Soc. Trop. Med. Hyg. 103, 390-394. doi: 10.1016/j.trstmh.2008.09.009

Li, R., Li, Y., Kristiansen, K., and Wang, J. (2008). SOAP: short oligonucleotide alignment program. Bioinformatics 24, 713-714. doi: 10.1093/bioinformatics/ btn025

Liu, Y. Y., Chen, C. C., and Chiou, C. S. (2016). Construction of a pan-genome allele database of Salmonella enterica serovar enteritidis for molecular subtyping and disease cluster identification. Front. Microbiol. 7:2010. doi: 10.3389/fmicb.2016. 02010

Liu, Y. Y., Lin, J. W., and Chen, C. C. (2019). Cano-wgMLST_BacCompare: a bacterial genome analysis platform for epidemiological investigation and comparative genomic analysis. Front. microbiol. 10:1687. doi: 10.3389/fmicb. 2019.01687

Lyu, N., Feng, Y. Q., Pan, Y. L., Huang, H., Liu, Y., Xue, C., et al. (2021). Genomic characterization of Salmonella enterica isolates from retail meat in Beijing, China. Front. Microbiol. 12:636332. doi: 10.3389/fmicb.2021.636332

Maiden, M. C. J., Bygraves, J. A., Feil, E., Morelli, G., Russell, J. E., Urwin, R., et al. (1998). Multilocus sequence typing: a portable approach to the identification of clones within populations of pathogenic microorganisms. Proc. Natl. Acad. Sci. U.S.A. 95, 3140-3145. doi: 10.1073/pnas.95.6.3140

Mairi, A., Barraud, O., Muggeo, A., de Champs, C., and Touati, A. (2020). Genomic analysis of a multidrug-resistant Klebsiella pneumoniae ST11 strain recovered from Barbary deer (Cervus elaphus barbarus) in Akfadou Forest, Algeria. J. Glob. Antimicrob. Resist. 22, 515-518. doi: 10.1016/j.jgar.2020. 04.027

Mellmann, A., Dag, H., Craig, A., Cummings, E. B., Zentz, S. R., Leopold, A. R., et al. (2011). Prospective genomic characterization of the German enterohemorrhagic escherichia coli $\mathrm{O} 104: \mathrm{H} 4$ outbreak by rapid next generation sequencing technology. PLoS One 6:e22751. doi: 10.1371/journal.pone.0022751

Mezal, E. H., Sabol, A., Khan, M. A., Ali, N., Stefanova, R., and Khan, A. A. (2014). Isolation and molecular characterization of Salmonella enterica serovar Enteritidis from poultry house and clinical samples during 2010. Food Microbiol. 38, 67-74. doi: 10.1016/j.fm.2013.08.003

Monte, D. F. M., Nethery, M. A., Barrangou, R., Landgraf, M., and Fedorka-Cray, P. J. (2021). Whole-genome sequencing analysis and CRISPR genotyping of rare antibiotic-resistant Salmonella enterica serovars isolated from food and related sources. Food Microbiol. 93:103601. doi: 10.1016/j.fm.2020.103601

Perry, F., Casey, J., Bridget, A., and Ryan, J. A. (2020). The differential phosphorylation-dependent signaling and glucose immunometabolic responses induced during infection by Salmonella enteritidis and Salmonella heidelberg in chicken macrophage-like cells. Microorganisms 8:1041. doi: 10.3390/ microorganisms 8071041

Possebon, F. S., Tiba Casas, M. R., Nero, L. A., Yamatogi, R. S., Araújo, J. P., Pinto, J. P., et al. (2020). Prevalence, antibiotic resistance, PFGE and MLST characterization of Salmonella in swine mesenteric lymph nodes. Prev. Vet. Med. 179:105024. doi: 10.1016/j.prevetmed.2020.105024

Quick, J., Ashton, P., Calus, S., Chatt, C., Gossain, S., Hawker, J., et al. (2015). Rapid draft sequencing and real-time nanopore sequencing in a hospital outbreak of Salmonella. Genome Biol. 16:114. doi: 10.1186/s13059-015-0 $677-2$

Radomski, N., Sabrina, C., Emeline, C., Arnaud, F., Pauline, B., Federica, P., et al. (2019). A simple and robust statistical method to define genetic relatedness of samples related to outbreaks at the genomic scale-application to retrospective Salmonella foodborne outbreak investigations. Front. Microbiol. 10:2413. doi: 10.3389/fmicb.2019.02413

Robertson, J., Yoshida, C., Kruczkiewicz, P., Nadon, C., Nichani, A., Taboada, E. N., et al. (2018). Comprehensive assessment of the quality of Salmonella whole genome sequence data available in public sequence databases using the Salmonella in silico Typing Resource (SISTR). Microb. Genom. 4:e000151. doi: 10.1099/mgen.0.000151

Robertson, S. A., Sidge, J. L., Koski, L., Hardy, M. C., Stevenson, L., Signs, K., et al. (2019). Onsite investigation at a mail-order hatchery following a multistate Salmonella illness outbreak linked to live poultry-United States, 2018. Poult. Sci. 98, 6964-6972. doi: 10.3382/ps/pez529

Ruppitsch, W., Ariane, P., Karola, P., Stefan, B., Haizpea, L. F., Franz, A., et al. (2015). Defining and evaluating a core genome multilocus sequence typing scheme for whole-genome sequence-based typing of Listeria monocytogenes. J. Clin. Microbiol. 53, 2869-2876. doi: 10.1128/JCM. 01193-15

Sabat, A. J., Sandra, M. H., Viktoria, A., Amadu, J., John, E. D., Hajo, G., et al. (2017). Complete-genome sequencing elucidates outbreak dynamics of ca-mrsa usa300 (st8-spa t008) in an academic hospital of Paramaribo, Republic of Suriname. Sci. Rep. 7:41050. doi: 10.1038/srep41050

Scornavacca, C., Zickmann, F., and Huson, D. H. (2011). Tanglegrams for rooted phylogenetic trees and networks. Bioinformatics 27, i248-i256. doi: 10.1093/ bioinformatics/btr210

Seif, Y., Kavvas, E., Lachance, J. C., Yurkovich, J. T., Nuccio, S. P., Fang, X., et al. (2018). Genome-scale metabolic reconstructions of multiple Salmonella strains reveal serovar-specific metabolic traits. Nat. Commun. 9:3771. doi: 10.1038/ s41467-018-06112-5

Shen, H., Chen, H., Ou, Y., Huang, T., Chen, S., Zhou, L., et al. (2020). Prevalence, serotypes, and antimicrobial resistance of Salmonella isolates from patients with diarrhea in Shenzhen, China. BMC Microbiol. 20:197. doi: 10.1186/s12866-02001886-5

Simon, S., Trost, E., Bender, J., Fuchs, S., Malorny, B., Rabsch, W., et al. (2018). Evaluation of WGS based approaches for investigating a food-borne outbreak caused by Salmonella enterica serovar Derby in Germany. Food Microbiol. 71, 46-54. doi: 10.1016/j.fm.2017.08.017

Simpson, J. T., Wong, K., Jackman, S. D., Schein, J. E., Jones, S. J. M., and Birol, I. (2009). ABySS: a parallel assembler for short read sequence data. Genome Res. 19, 1117-1123. doi: 10.1101/gr.089532.108

Tiba-Casas, M. R., Cláudio, T. S., Cláudia, R. G., Elisabete, A. A., Flávia, B. S., Amanda Maria de, J. B., et al. (2019). Molecular analysis of clonally related Salmonella Typhi recovered from epidemiologically unrelated cases of typhoid fever, Brazil. Int. J. Infect. Dis. 81, 191-195. doi: 10.1016/j.ijid.2019. 02.009

Uelze, L., Borowiak, M., Deneke, C., Szabó, I., Fischer, J., Tausch, S. H., et al. (2019). Comparative assessment of the performance and accuracy of four open-source tools for in silico serotyping of Salmonella spp. based on whole-genome short read sequencing data. Appl. Environ. Microbiol. 86:e2265-19. doi: 10.1128/AEM. 02265-19

Vincent, C., Usongo, V., Berry, C., Tremblay, D. M., Moineau, S., Yous, K., et al. (2018). Comparison of advanced whole genome sequence-based methods to distinguish strains of Salmonella enterica serovar Heidelberg involved in foodborne outbreaks in Québec. Food Microbiol. 73, 99-110. doi: 10.1016/j.fm. 2018.01.004

Wang, M., Zhang, M., Lu, Y., Kang, X., Meng, C., Zhou, L., et al. (2020). Analyses of prevalence and molecular typing of Salmonella in the goose production chain. Poult. Sci. 99, 2136-2145. doi: 10.1016/j.psj.2019.12.008

Wang, X., Wang, H., Li, T., Liu, F., Cheng, Y., Guo, X., et al. (2020). Characterization of Salmonella spp. isolated from chickens in Central China. BMC Vet. Res. 16:299. doi: 10.1186/s12917-020-02513-1 
Wang, Y. J., Zhang, L. H., Niu, T., and Feng, Y. (2020). Genomic characterization of a multidrug-resistant Salmonella enterica serovar goldcoast sequence type 358 strain in China. J. Glob. Antimicrob. Resist. 23, 181-183. doi: 10.1016/j.jgar. 2020.09.007

World Health Organization (WHO). (2003). Basic Laboratory Procedures in Clinical Bacteriology, 2nd Edn. Geneva: World Health Organization, 37-50.

Xin, Y., Zhu, H. W., Bo, Y. H., Li, Y. Z., Zhang, Y., Liu, Y., et al. (2021). Prevalence and antimicrobial resistance of Salmonella enterica subspecies enterica serovar Enteritidis isolated from broiler chickens in shandong province. China, 20132018. Poult. Sci. 100, 1016-1023. doi: 10.1016/j.psj.2020.09.079

Yang, X., Wu, Q., Zhang, J., Huang, J., Chen, L., Wu, S., et al. (2019). Prevalence, bacterial load, and antimicrobial resistance of Salmonella serovars isolated from retail meat and meat products in China. Front. Microbiol. 10:2121. doi: 10.3389/ fmicb.2019.02121

Yin, Z., Liu, J., Du, B., Ruan, H. H., Huo, Y. X., Du, Y., et al. (2020). Whole-genomebased survey for polyphyletic serovars of Salmonella enterica subsp. enterica provides new insights into public health surveillance. Int. J. Mol. Sci. 21:5226. doi: 10.3390/ijms21155226

Yoshida, C. E., Kruczkiewicz, P., Laing, C. R., Lingohr, E. J., Gannon, V. P. J., Nash, J. H. E., et al. (2016). The Salmonella in silico typing resource (SISTR): an open web-accessible tool for rapidly typing and subtyping draft Salmonella genome assemblies. PLoS One 11:e0147101. doi: 10.1371/journal.pone.014 7101

Zhang, S., den Bakker, H. C., Li, S., Chen, J., Dinsmore, B. A., Lane, C., et al. (2019). SeqSero2: rapid and improved Salmonella serotype determination using whole-genome sequencing data. Appl. Environ. Microbiol. 85:e01746-19. doi: 10.1128/AEM.01746-19

Zhang, S., Yin, Y., Jones, M. B., Zhang, Z., Kaiser, B. L. D., Dinsmore, B. A., et al. (2015). Salmonella serotype determination utilizing high-throughput genome sequencing data. J. Clin. Microbiol. 53, 1685-1692. doi: 10.1128/JCM.00323-15
Zhang, Z., Yang, J., Xu, X., Zhou, X., Shi, C., Zhao, X., et al. (2020). Co-existence of mphA, oqXAB and blaCTX-M-65 on the IncHI2 Plasmid in highly drugresistant Salmonella enterica serovar Indiana ST17 isolated from retail foods and humans in China. Food Control 118:107269. doi: 10.1016/j.foodcont.2020. 107269

Zhao, X., Hu, M., Zhang, Q., Zhao, C., Zhang, Y., Li, L., et al. (2020). Characterization of integrons and antimicrobial resistance in Salmonella from broilers in Shandong, China. Poult. Sci. 99, 7046-7054. doi: 10.1016/j.psj.2020. 09.071

Zhou, Z., Alikhan, N. F., Mohamed, K., Fan, Y., and Achtman, M. (2020). The EnteroBase user's guide, with case studies on Salmonella transmissions, Yersinia pestis phylogeny, and Escherichia core genomic diversity. Genome Res. 30, 138-152. doi: 10.1101/gr.251678.119

Conflict of Interest: The authors declare that the research was conducted in the absence of any commercial or financial relationships that could be construed as a potential conflict of interest.

Publisher's Note: All claims expressed in this article are solely those of the authors and do not necessarily represent those of their affiliated organizations, or those of the publisher, the editors and the reviewers. Any product that may be evaluated in this article, or claim that may be made by its manufacturer, is not guaranteed or endorsed by the publisher.

Copyright (c) 2021 Yan, Zhang, Li, Liu, Zhu, Chen and Yang. This is an open-access article distributed under the terms of the Creative Commons Attribution License (CC BY). The use, distribution or reproduction in other forums is permitted, provided the original author(s) and the copyright owner(s) are credited and that the original publication in this journal is cited, in accordance with accepted academic practice. No use, distribution or reproduction is permitted which does not comply with these terms. 